\title{
The SUPERankle procedure in the treatment of foot and ankle deformities in fibular hemimelia
}

\author{
Carlos Eduardo Cabral Fraga' ${ }^{(\mathbb{D})}$, Gabriel João Francisco Souza' ${ }^{\mathbb{D}}$, Jefferson Soares Martins' ${ }^{(\mathbb{D})}$, \\ Marcos Vinicius Muniz Lemos Souto ${ }^{\mathbb{D}}$, Rodolfo Amoedo de Castilho Candido Pinto' ${ }^{\mathbb{D}}$, Túlio Romano Troncoso Chaves ${ }^{1(\mathbb{D})}$ \\ 1. Hospital das Clínicas da Universidade Federal de Goiás, Goiânia, Goiás, Brazil.
}

\begin{abstract}
Objective: To review indications for limb amputation or reconstruction using the SUPERankle procedure in patients diagnosed with Paley type 3 or 4 fibular hemimelia; To evaluate the correction of deformities needed to achieve a stable plantigrade foot, review variations of the original technique and their applicability, and describe challenges encountered in their execution.

Methods: Qualitative, retrospective, descriptive study of 4 patients who underwent the SUPERankle procedure between 2019 and 2020 for treatment of foot and ankle deformities in fibular hemimelia. Pre- and postoperative clinical and radiographic evaluations were performed to identify objective evidence of the correction of foot and ankle deformities. The operative techniques employed are described.

Results: The included patients met anatomical and psychosocial criteria for reconstruction, as established in the literature. Clinical correction of foot and ankle deformities was achieved, but on radiographic evaluation, there was no statistical difference; this was likely due to the small number of patients, given the rarity of fibular hemimelia.

Conclusion: The procedure is reproducible, but requires a knowledgeable, well-trained surgical team. Correction of foot and ankle deformities can be achieved. The choice between amputation or reconstruction should be the result of an informed decision by family members, supported by a multidisciplinary team.
\end{abstract}

Level of Evidence IV; Qualitative Study; Case Series.

Keywords: Hemimelia; Fibula; Reconstructive surgical procedures; Leg length inequality.

\section{Introduction}

Fibular hemimelia $(\mathrm{FH})$ is part of a broad spectrum of lower-limb deformities that include partial or total absence of the fibula, associated with other abnormalities (Figure 1), including reduced length of the affected limb, angular deformities, ball-and-socket ankle joint, tarsal anomalies, absent lateral rays of the foot, short femur, and occasionally, hand anomalies ${ }^{(1-4)}$.

It is a rare deformity, with an incidence ranging from 7.4 to 49 cases per $1,000,000$ live births ${ }^{(1,4,5)}$. The etiology is still unknown, and most cases occur in the absence of a family history of birth defects. Bilateral HF is the exception, as it often represents an autosomal dominant condition ${ }^{(2)}$. The classification described by Achterman and Kalamchi ${ }^{(6)}$ in 1979 divided $\mathrm{FH}$ into 2 types, according to its anatomical and radiographic characteristics ${ }^{(1,6,7)}$. More recently, Paley's classification became the first one developed specifically to provide surgical options for reconstruction ${ }^{(1)}$. This classification is based on clinical examination and plain radiographs of the foot and ankle. If there is no foot deformity and the ankle is stable, the condition is Paley type 1. If the ankle deformity is dynamic, it is type 2. If there is a fixed equinovalgus deformity, it is type 3. In type 3A, the deformity is located in the ankle; in type 3B, it is subtalar; and in type 3C, it is combined ankle and subtalar. In type 4 , there is a fixed equinovarus deformity. MRI is not required to distinguish types $1,2,3$, and 4 , but it helps subdivide type 3 into $A, B$, or $C^{(2)}$.
Study performed at the Hospital das Clínicas da Universidade Federal de Goiás, Goiânia, Goiás, Brazil.

Correspondence: Túlio Romano Troncoso Chaves. Rua R-8, no 22, Setor Oeste - 74125-130, Goiânia, Goiás, Brazil. E-mail: tuliortc@gmail.com. Conflicts of Interest: none. Source of funding: none. Date received: July 09, 2021. Date accepted: August 14, 2021. Online: December 20, 2021.
How to cite this article: Fraga CEC, Souza GJF, Martins JS, Souto MVML, Pinto RACC, Chaves TRT. The SUPERankle procedure in the treatment of foot and ankle deformities in fibular hemimelia. J Foot Ankle. 2021;15(3):217-22. 

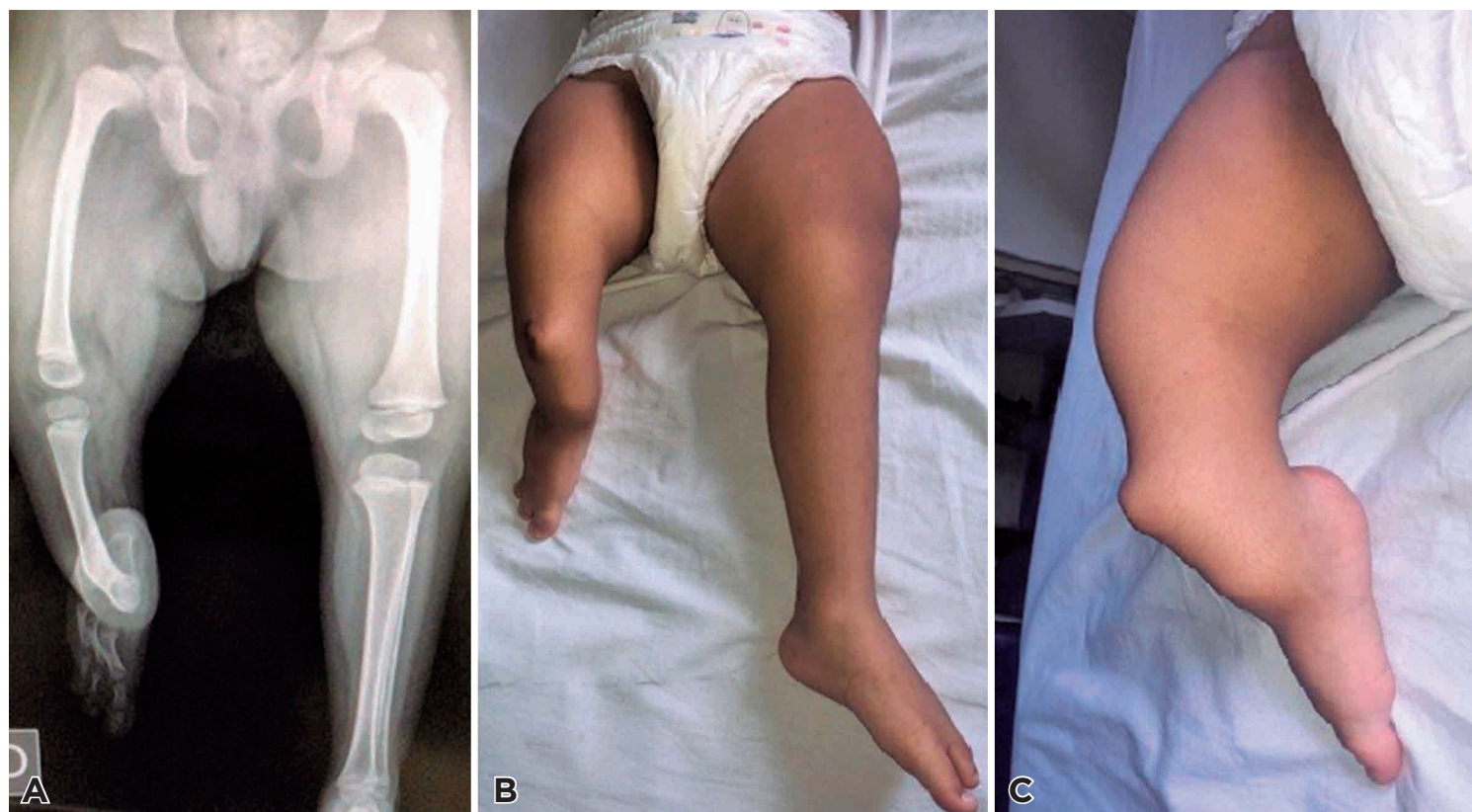

Figure 1. A) Plain radiograph of lower limbs. B) Clinical picture (anteroposterior view). C) Clinical picture (lateral view).

Syme or Boyd amputations were long the only treatment option, and are still indicated by several authors and used to good effect; however, advances in bone reconstruction have enabled an alternative to amputation ${ }^{(8,9)}$. The SUPERankle procedure, SUPER standing for Systematic Utilitarian Procedure for Extremity Reconstruction, is the term used by Paley ${ }^{(2)}$ to describe a systematic procedure for ankle and foot extremity reconstruction. A combination of surgical approaches to bone and soft tissues, stabilizing the foot and correcting the associated deformities, it is indicated for the treatment of Paley type 3 and $4 \mathrm{FH}^{(2)}$.

The present study was designed to obtain clinical and radiographic evidence of correction of foot and ankle deformities in 4 patients who underwent the SUPERankle procedure, as well as to analyze the reproducibility of the original technique and its variations, describe the surgical procedure, and discuss in detail the technical challenges encountered.

\section{Methods}

This study was approved by the local ethics committee and registered on Plataforma Brasil. Four patients (Table 1) diagnosed with Paley type 3 or $4 \mathrm{FH}$ underwent reconstructive treatment with the SUPERankle procedure between January 2019 and February 2020. All procedures were performed by the same team.

Patients were evaluated for descriptive variables, age, sex, affected side, associated deformities, and previous surgeries. To evaluate surgical correction, all cases underwent detailed radiographic evaluation before and after surgery, where we measured the talocalcaneal, tibiotalar, and plantigrade angles of the foot (Figure 2) on lateral views of the foot and ankle. The talocalcaneal angle is calculated by tracing lines through the axis of the talar neck and the long axis of the calcaneus. The tibiotalar angle is calculated by tracing lines through the axis of the talar neck and the anatomical axis of the tibia. Finally, the plantigrade angle of the foot is the angle between the anatomical axis of the tibia and the load-bearing surface of the foot ${ }^{(10)}$. These angles were compared with normal values described in the literature ${ }^{(10)}$ (Table 2), and the mean angles before and after surgical treatment were calculated. The degree of satisfaction of patients and their families was evaluated dichotomously as "satisfied" or "dissatisfied" with the surgery.

\section{Surgical technique}

The patient was placed in supine position with a 20-degree lateral positioning on the operated side. The whole procedure is performed under pneumatic tourniquet control.

Step 1: A longitudinal incision was made on the lateral aspect of the leg, with layered dissection to avoid injury to the sural nerve.

Step 2: The lateral structures were identified; when both the peroneus brevis and longus tendons are present, the brevis was lengthened by z-plasty. Three patients had only the peroneus longus, which did not need to be lengthened, as it was not tense nor prevented subtalar reduction.

Step 3: The cartilaginous fibular anlage (Figure 3), which corresponds to the fibular remnant(2), was identified. This structure can range from a soft, fibrous cord to a dysplastic fibula with structured bone tissue. In older patients, the anlage is more evident. In three patients, it was adherent to 
Table 1. Data on patients who underwent the SUPERankle procedure

\begin{tabular}{|c|c|c|c|c|c|c|}
\hline & Sex & Paley type & Age & Side & Associated deformities & Previous surgery \\
\hline Patient 1 & $\mathrm{~F}$ & 3B & 8 years & $\mathrm{L}$ & Short femur & Yes \\
\hline Patient 2 & M & $3 \mathrm{~A}$ & 7 years & $\mathrm{R}$ & $\begin{array}{c}\text { Short femur } \\
\text { Absence of single ray }\end{array}$ & No \\
\hline Patient 3 & $\mathrm{~F}$ & $3 C$ & 8 years & $\mathrm{R}$ & $\begin{array}{c}\text { Short femur } \\
\text { Absence of single ray }\end{array}$ & Yes \\
\hline Patient 4 & M & $3 B$ & 4 years & $\mathrm{R}$ & $\begin{array}{c}\text { Short femur } \\
\text { Absence of two rays }\end{array}$ & No \\
\hline
\end{tabular}
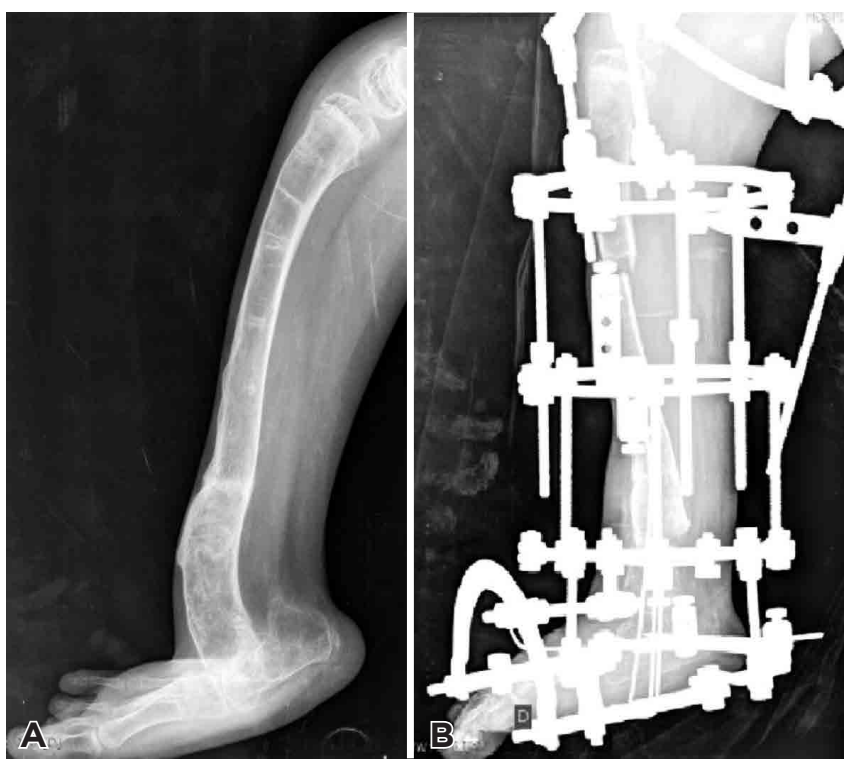

Figure 2. A) Preoperative radiograph. B) Postoperative radiograph.

Table 2. Reference values of angles

\begin{tabular}{lc}
\hline Lateral talocalcaneal angle & $35-50$ \\
Tibiotalar angle & $68(64-72)$ \\
Plantigrade angle of the foot & $88(85-91)$ \\
\hline
\end{tabular}

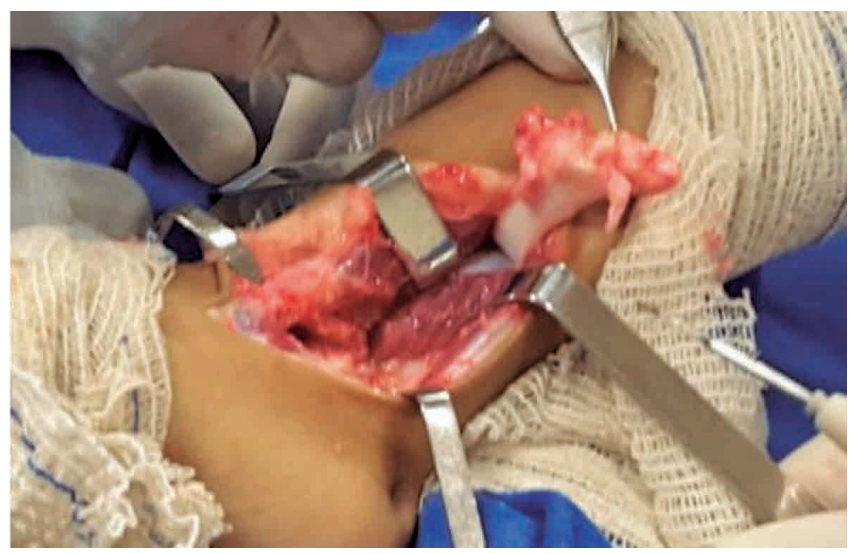

Figure 3. Fibular anlage. the lateral aspect of the calcaneus, and was dissected and removed with a scalpel and a delicate osteotome. Unlike in the technique originally described by Paley, we performed the lateral incision only distally. We achieved good resection of the cartilaginous anlage and dysplastic fibula through this distal incision, with no need to extend the incision proximally.

Step 4: After resection of the fibular anlage, the flexor hallucis and neurovascular structures were identified, and the neurovascular bundle dissected and decompressed.

Step 5: The lateral wall of the calcaneus was then cleaned, and the sinus tarsi and posterior edge of the calcaneus identified. A lateral capsulotomy was performed, and the ankle and subtalar joints were identified. The cartilaginous talocalcaneal junction was identified and the osteotome advanced at a $45^{\circ}$ angle to cut through the subtalar coalition, starting the cut posteriorly and moving towards the sinus tarsi.

Step 6: After subtalar release, the calcaneus was reduced by medializing it and bringing it under the talus, correcting the hindfoot valgus, and pinning the calcaneus, talus, and tibia with two $2.0 \mathrm{~K}$-wires.

Step 7: A T-shaped incision was made in the periosteum of the distal tibia. Two guide wires were inserted parallel to the plantar surface in the frontal and sagittal planes, and a distal osteotomy was made parallel to the guide wires with a saw.

Step 8: After tibial osteotomy, the distal segment was shifted medially to overlap the tibial bone ends. The level of the overlap was marked. Guide wires were inserted at the level of the overlap, which is the site of the second shortening osteotomy. This osteotomy was made perpendicularly to the axis of the proximal diaphysis of the tibia, forming a trapezoidal-shaped piece of bone to be resected (Figure 4). If there is procurvatum angulation or diaphyseal deformity, the second osteotomy can be distal to or at the apex of this deformity. This second osteotomy is performed to straighten and shorten the tibia. In naturally shorter tibias, this shortening of the limb hinders later assembly of the llizarov frame.

Step 9: After the guide wires and bone segment were removed, the tibia was realigned and shortened. The retrograde axial K-wires used for subtalar fixation were advanced up the tibia (Figure 5). If the cuts were performed correctly, the foot should be plantigrade at this point. In one case, we used three $2.0 \mathrm{~K}$-wires. The skin over the anterior distal tibia can be dissected to avoid creating an anterior skin fold. Hemostasis is then achieved and the lateral incision closed in layers. The foot is plantigrade for assembly of the external ring fixator. 


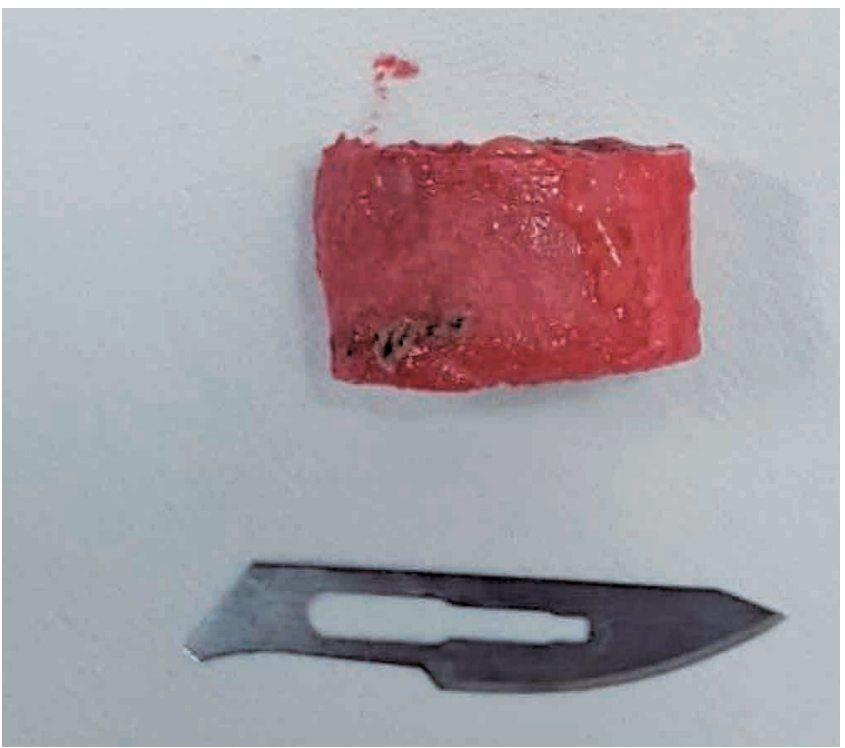

Figure 4. Tibial osteotomy.

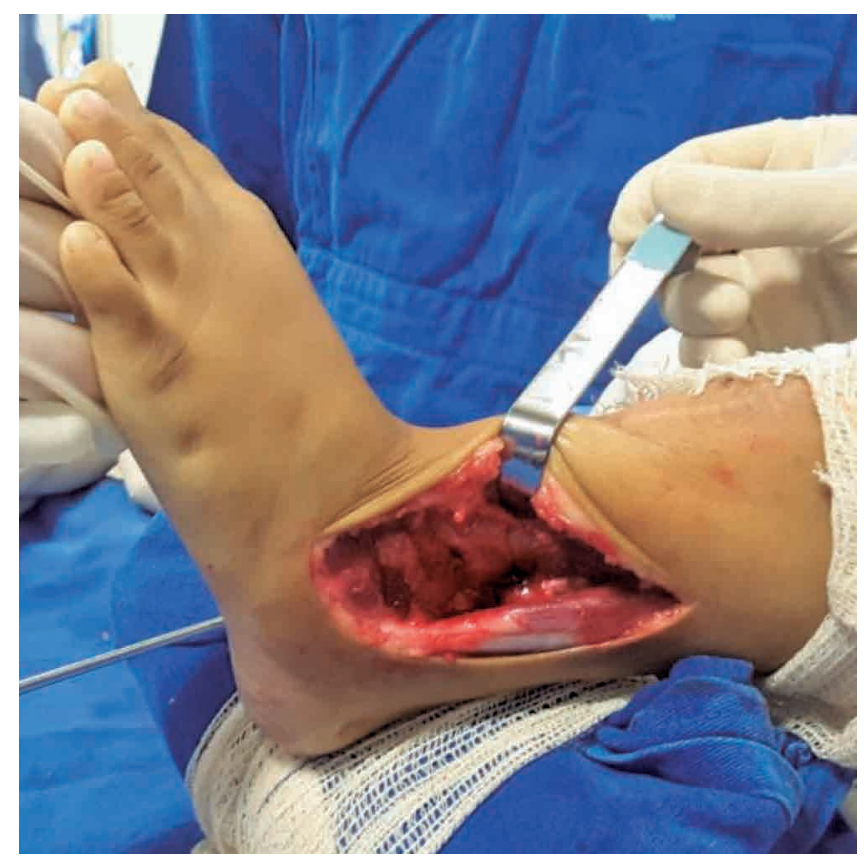

Figure 5. Fragment reduction and retrograde wire fixation.

Step 10: A traditional Ilizarov external ring fixator was placed. The assembly consists of a tibial block with two or three rings depending on the length of the tibia. The foot assembly is then applied, fixing the hindfoot and forefoot in plantigrade position. Schanz pins are used to fix the calcaneus, and smooth wires to fix the forefoot.
Step 11: The femoral block was assembled with an arch and a ring, which were connected with struts to the tibial block. Addition of the femoral assembly is necessary due to knee instability caused by agenesis of the cruciate ligaments in these cases.

Step 12: After assembly of the external fixator, tibial osteotomy for lengthening was performed through an anteromedial approach in the proximal third of the leg. Finally, the skin was sutured and the wound was dressed.

\section{Results}

The mean age was 6.7 years (range, $4-8$ years); $50 \%(n=2)$ were female and $50 \%(n=2)$ were male. There was a predominance of right-sided involvement. Two patients had previously undergone surgery on the affected limb (Table 1).

Results regarding angular correction of foot and ankle deformities, as determined by comparison of pre- and postoperative period radiographs, are shown in Table 3. There was a trend toward near-normal angular values after surgery, as shown in Table 2.

The mean and standard deviation of the angles obtained after surgery were compared with the normal values using the one-sample $T$-test. P-values $<0.05$ were considered significant. By this measure, there was no significant difference between postoperative angular values and normal values (Table 4). Statistical analyses were performed in SPSS Statistics for Windows, Version 20.0 (Armonk, NY: IBM Corp). In terms of satisfaction with the procedure, all patients and family members were satisfied with the outcome of treatment. In all patients, clinical correction of the deformity was observed, with a stable plantigrade foot that can bear weight for walking and provide support for subsequent reconstruction procedures (Figure 6).

\section{Discussion}

The SUPERankle procedure for reconstruction in fibular hemimelia, devised by Paley, uses a hexapod external fixator. This system uses the 6 -axis correction principle, guided by computer software. The tibial ring block assembly uses only two rings, the reference (or stationary) ring and the mobility ring.

In this study, we used the llizarov ring fixator, as it is the only circular fixator available at our facility. This external fixator offers a smaller working area after placement of the rings in

Table 3. Pre- and postoperative angles

\begin{tabular}{|c|c|c|c|c|c|c|}
\hline & \multicolumn{2}{|c|}{ Talocalcaneal } & \multicolumn{2}{|c|}{ Thbiotalar } & \multicolumn{2}{|c|}{ Plantigrade } \\
\hline & Pre & Post & Pre & Post & Pre & Post \\
\hline Patient 1 & 20.1 & 50 & 57.4 & 72.1 & 114.2 & 89.9 \\
\hline Patient 2 & 18.6 & 44.5 & 59.4 & 81.4 & 81.4 & 88 \\
\hline Patient 3 & 18.3 & 25.8 & 24 & 66 & 66 & 85 \\
\hline Patient 4 & 14 & 23 & 58.1 & 74 & 57.5 & 78.9 \\
\hline
\end{tabular}


Table 4. Mean (standard deviation) postoperative angles and their reference ranges

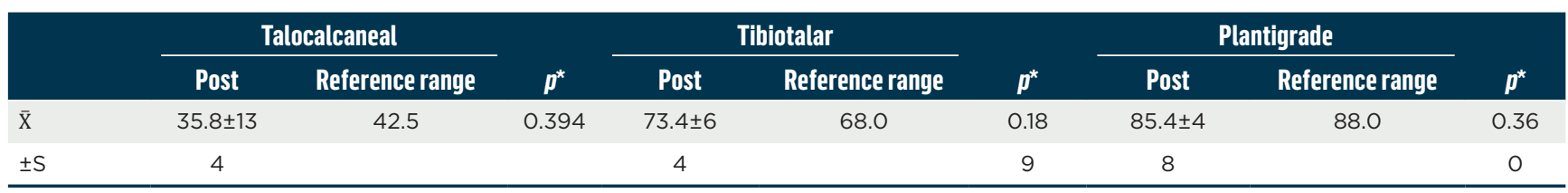

$\overline{\mathrm{X}}$ : mean. S: standard deviation. *One-sample $T$-test.

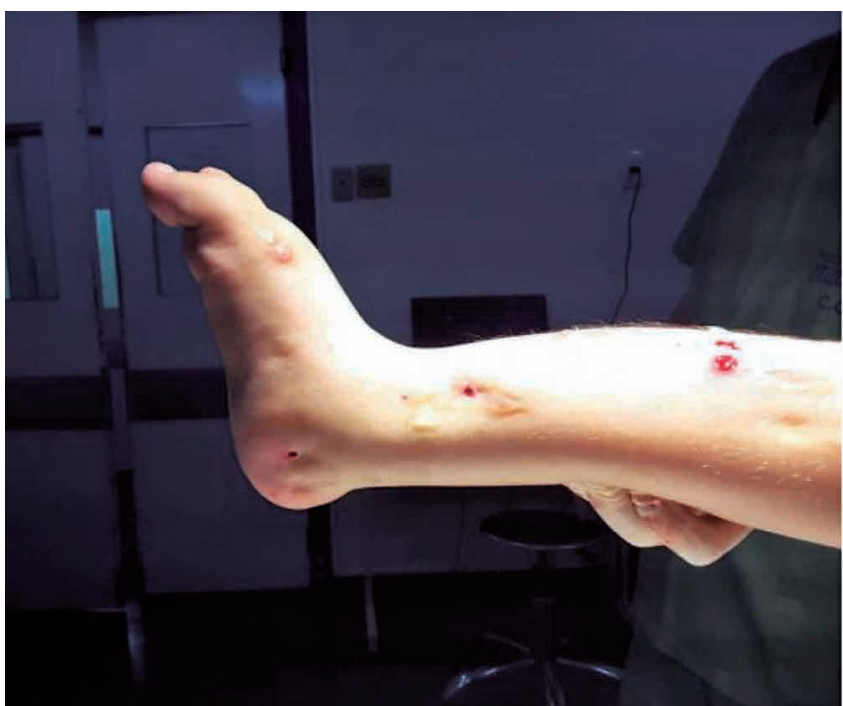

Figure 6. Clinical picture after removal of the external fixator.

the tibial assembly, causing greater difficulty in managing the proximal tibial osteotomy to initiate bone lengthening during the same surgical stage of the SUPERankle procedure. This technical difficulty was also reported by Alaseirlis et al. ${ }^{(11)}$.

We believe that a conventional external ring fixator is suitable for performing the SUPERankle procedure, and if technical difficulty in performing the proximal tibial osteotomy is encountered, lengthening can be performed later. The same conclusion was reached by Kulkarni et al. ${ }^{(12)}$, who recommend that lengthening be performed 1 or 2 years after the SUPERankle procedure.

Paley advocates that treatment of fibular hemimelia should preferably be carried out between 18 and 24 months of age. When performed after 5 years of age, there is a greater chance of recurrence of deformities and joint stiffness in the ankle ${ }^{(12)}$. Three patients in the present study only had access to surgery at such relatively advanced ages. The indication for limb reconstruction should be maintained, and future recurrence of deformities, if any, should be corrected during treatment. Major residual deformities include lower limb length discrepancy, calf atrophy, and ankle valgus ${ }^{(13)}$. All patients in this study will remain under follow-up by the attending medical team to correct any associated deformities, obtain a stable limb, and ensure no length discrepancies remain once they reach skeletal maturity.
Patients 1 and 3 had already undergone previous surgeries for lengthening and correction of the tibial deformity, but unsuccessfully, as a plantigrade foot had not been achieved. The success of limb reconstruction treatment in patients with Paley type 3 and $4 \mathrm{FH}$ depends on the specific approach to these foot and ankle deformities.

All patients included herein had at least three rays in the foot and, as proposed by Birch et al. ${ }^{(14)}$, would thus be candidates for limb reconstruction as an alternative to amputation. This criterion-presence of at least three rays-is indispensable to obtaining a plantigrade, stable foot for ambulation.

We did not any find data in the literature regarding radiological evaluation of foot and ankle angles in patients undergoing the SUPERankle procedure for the treatment of $\mathrm{FH}$. We believe that measuring these angles provides objective criteria for evaluating the correction of foot and ankle deformities. Although we found a tendency toward near-normal angular values, there was no significant difference, probably due to the small number of patients, which, in turn, is expected given the rare nature of this deformity.

Calder et al. ${ }^{(15)}$ suggest that an expected discrepancy greater than $20 \mathrm{~cm}$ would be a formal indication for amputation, due to the need for more than three lengthening procedures or epiphysiodesis of the normal limb. In none of the cases presented herein would the shortening predicted by the Multiplier method(16) upon reaching skeletal maturity exceed 20 centimeters.

The external fixator was removed an average of 11 to 12 months after the initial procedure, and tibial lengthening was performed to approximately 4 to 5 centimeters on average. This is within the expected range, as all patients will undergo 2 more lengthening procedures during skeletal maturation. Lengthening is performed at home, with guidance to family members regarding proper handling of the device. Outpatient visits took place every other week in the first 3 months and monthly thereafter until the fixator was removed.

Another factor to be analyzed when deciding between early amputation or correction of deformities and limb growth discrepancy are the psychological consequences of such procedures. Studies show that no significant differences were found in psychological adjustment and quality of life when comparing amputation and reconstruction in patients with $\mathrm{FH}^{(8)}$. Patients undergoing Syme amputation have good psychological and functional outcomes(17).

Moraal et al. ${ }^{(7)}$ state that patients undergoing serial reconstruction procedures with prolonged use of the llizarov techni- 
que experience improvement in self-esteem after correction of the deformity and limb discrepancy. A decrease in pain and a greater degree of satisfaction were also found after correction of the length discrepancy, with better psychological and satisfaction outcomes. In the present study, all family members showed interest and were $100 \%$ satisfied with the outcome of reconstructive treatment. If they decide to interrupt reconstruction of the affected limb and undergo amputation, the procedure can still be performed by the team.

White ethnicity, higher education, and higher family income are associated with choosing reconstructive treatment ${ }^{(8)}$. The findings of the present study are not consistent with the international literature. Of the four patients whose families chose reconstruction surgery, three are Black and one White; all come from low-income, low-education background.
All patients are being actively followed, with the expectation of undergoing an additional lengthening procedure 3 to 4 years after the first procedure. During this period, they will undergo serial clinical and radiological examinations to screen for recurrence of deformities or other complications.

\section{Conclusion}

SUPERankle reconstruction surgery requires a team of operators knowledgeable in foot and ankle anatomy and familiar with external fixation techniques. In such hands, it is reproducible and achieves good clinical and radiological outcomes. In all cases presented herein, correction of foot and ankle deformities was achieved, obtaining a plantigrade foot which can provide stability and function to the limb after reconstruction.

Authors' contributions: Each author contributed individually and significantly to the development of this article: CECF *(https://orcid.org/OOOO-OOO23832-7397) Conceived and planned the activities that led to the study, interpreted the results of the study, data collection, participated in the review process, performed the surgeries, clinical examination and approved the final version; GJFS *(https://orcid.org/0000-0001-8490-6085) Conceived and planned the activities that led to the study, interpreted the results of the study, participated in the review process and approved the final version; JSM *(https://orcid.org/0000-0003-4742-1905) Conceived and planned the activities that led to the study, interpreted the results of the study, participated in the review process, performed the surgeries, clinical examination and approved the final version, formatting of the article, bibliographic review and approved the final version; MVMLS *(https://orcid.org/0000-0003-1003-7328) Conceived and planned the activities that led to the study, interpreted the results of the study, participated in the review process, statistical analysis and approved the final version; RACCP *(https://orcid.org/0000-0001-7434-1279) Conceived and planned the activities that led to the study, interpreted the results of the study, participated in the review process, performed the surgeries, clinical examination and approved the final version; TRTC *(https://orcid.org/0000-0003-2772-4802) Conceived and planned the activities that led to the study, interpreted the results of the study, participated in the review process, performed the surgeries, clinical examination and approved the final version, formatting of the article, bibliographic review and approved the final version. All authors read and approved the final manuscript. *ORCID (Open Researcher and Contributor ID) (iD).

\section{References}

1. Zhang Z, Yi D, Xie R, Hamilton JL, Kang QL, Chen D. Postaxial limb hypoplasia (PALH): the classification, clinical features, and related developmental biology. Ann N Y Acad Sci. 2017;1409(1):67-78.

2. Paley D. Surgical reconstruction for fibular hemimelia. J Child Orthop. 2016;10(6):557-83.

3. Catagni MA, Radwan M, Lovisetti L, Guerreschi F, Elmoghazy NA. Limb lengthening and deformity correction by the Ilizarov technique in type III fibular hemimelia: an alternative to amputation. Clin Orthop Relat Res. 2011;469(4):1175-80.

4. Oberc A, Sułko J. Fibular hemimelia - diagnostic management, principles, and results of treatment. J Pediatr Orthop B. 2013; 22(5):450-6

5. Froster UG, Baird PA. Congenital defects of lower limbs and associated malformations: a population based study. Am J Med Genet. 1993;45(1):60-4.

6. Achterman C, Kalamchi A. Congenital deficiency of the fibula. J Bone Joint Surg Br. 1979;61-B(2):133-7

7. Moraal JM, Elzinga-Plomp A, Jongmans MJ, Roermund PM, Flikweert $\mathrm{PE}$, Castelein RM, et al. Long-term psychosocial functioning after Ilizarov limb lengthening during childhood. Acta Orthop. 2009; 80(6):704-10.

8. Birch JG, Paley D, Herzenberg JE, Morton A, Ward S, Riddle R, et al. Amputation Versus Staged Reconstruction for Severe Fibular Hemimelia: Assessment of Psychosocial and Quality-of-Life Status and Physical Functioning in Childhood. JB JS Open Access. 2019;4(2):e0053.

9. Rogala EJ, Wynne-Davies R, Littlejohn A, Gormley J. Congenital limb anomalies:frequency and aetiological factors. Data from the Edinburgh Register of the Newborn (1964-68). J Med Genet. $1974 ; 11(3): 221-33$.
10. Lamm BM, Stasko PA, Gesheff MG, Bhave A. Normal Foot and Ankle Radiographic Angles, Measurements, and Reference Points. J Foot Ankle Surg. 2016;55(5):991-8.

11. Alaseirlis DA, Korompilias AV, Beris AE, Soucacos PN. Residual malformations and leg length discrepancy after treatment of fibular hemimelia. J Orthop Surg Res. 2011;6:51.

12. Kulkarni RM, Arora N, Saxena S, Kulkarni SM, Saini Y, Negandhi R. Use of Paley Classification and SUPERankle Procedure in the Management of Fibular Hemimelia. J Pediatr Orthop. 2019;39(9): e708-e717.

13. Unprasert P, Kaewpornsawan K, Chotigavanichaya C, Eamsobhana P. Management of fibular hemimelia using the llizarov method at Siriraj Hospital in Thailand. J Med Assoc Thai. 2014;97 Suppl 9:S44-S49.

14. Birch JG, Lincoln TL, Mack PW, Birch CM. Congenital fibular deficiency: a review of thirty years' experience at one institution and a proposed classification system based on clinical deformity. J Bone Joint Surg Am. 2011;93(12):1144-51.

15. Calder P, Shaw S, Roberts A, Tennant S, Sedki I, Hanspal R, et al. A comparison of functional outcome between amputation and extension prosthesis in the treatment of congenital absence of the fibula with severe limb deformity. J Child Orthop. 2017;11(4):318-25.

16. Paley D, Bhave A, Herzenberg JE, Bowen JR. Multiplier method for predicting limb-length discrepancy. J Bone Joint Surg Am. 2000; 82(10):1432-46

17. Birch JG, Walsh SJ, Small JM, Morton A, Koch KD, Smith C, et al. Syme amputation for the treatment of fibular deficiency. An evaluation of long-term physical and psychological functional status. J Bone Joint Surg Am. 1999;81(11):1511-8. 